\section{Kicking RCC in the GLUT}

\section{By Tim Fulmer, Senior Writer}

A Stanford University School of Medicine team has identified a compound that blocked the growth of renal cell carcinomas in mice by inhibiting GLUT1, a protein involved in a metabolic pathway unique to the tumors. ${ }^{1}$ The inhibitor is exclusively licensed to Ruga Corp., which now is optimizing the compound.

Renal cell carcinoma (RCC) is the most common type of kidney cancer in adults. The disease often is refractory to chemotherapy and radiotherapy, and as a result treatment usually involves surgical removal of all or part of the kidney.

The Stanford researchers, led by Amato Giaccia, a professor of radiation and cancer biology, decided to focus on a highly prevalent subset of renal tumors that have an inactivation of the von Hippel-Lindau tumor suppressor $(v H L)$ gene.

$v H L$ inactivation occurs in about $75 \%$ of RCCs and leads to aberrant upregulation of multiple proteins associated with cancer cell metabolism under hypoxic conditions, including VEGF, erythropoietin (EPO) and solute carrier family 2 facilitated glucose transporter member 1 (SLC2A1; GLUT1) ${ }^{2,3}$

The team started by setting up an in vitro screen to identify compounds that selectively blocked metabolic pathways in $v H L$-deficient RCC tumors without impairing the metabolism of healthy cells.

The screen of more than 64,000 molecules from Stanford's small molecule library identified two classes of molecules that were toxic to $v H L$-deficient RCC cells but nontoxic to cells with functional $v H L$.

One class-pyridyl anilino thiazoles-was described in a 2008 paper in Cancer Cell and shown to induce cell death in $v H L$-deficient RCCs. ${ }^{4}$

In the new work, a second class of compounds was shown to induce cell death in $v H L$-deficient RCC cells by blocking cellular glucose uptake via GLUT1. The GLUT1-targeting compounds did not trigger cell death in normal cells expressing wild-type $v H L$.

In mice with RCC xenografts, daily treatment with the lead GLUT1 inhibitor decreased tumor glucose uptake and significantly delayed tumor growth compared with vehicle treatment $(p<0.01)$. The compound showed no toxicity in normal tissues.
Data were published in Science Translational Medicine.

A patent covering the GLUT1 inhibitors described in the new paper has been exclusively licensed by Ruga, which "has made great progress in optimizing these molecules and advancing this class of compounds in preclinical development," Ruga president and CEO Ray Tabibiazar told SciBX. "The plan is to start studying the compounds' safety and efficacy profiles in the clinic sometime next year."

According to Tabibiazar, "An implication of the latest paper is that one could use GLUT1 expression profiles and/or $v H L$ genotyping as companion diagnostics to stratify RCC patients for receiving a GLUT1 inhibitor."

The findings "add to the rapidly growing body of data indicating that rationally designed attacks on tumor-specific metabolism can be effective and potentially free of many of the weaknesses of traditional chemotherapy," said Paul Bingham, VP of research at cancer metabolism company Cornerstone Pharmaceuticals Inc.

Cornerstone is focused on identifying compounds that disrupt glucose metabolism in cancer cells. The company's lead compound, CPI-613, is a small molecule that targets the mitochondrial enzymes pyruvate dehydrogenase (PDH) and $\alpha$-ketoglutarate dehydrogenase. The molecule is in Phase I/II testing to treat pancreatic cancer and in Phase I testing to treat hematologic malignancies.

Meanwhile, corresponding author Giaccia said his lab plans to look at the GLUT1 inhibitors in other tumor types. Tabibiazar suggested that "ovarian, lung, liver and colon cancers also might benefit from GLUT1 inhibition." Glucose metabolism is dysregulated in subtypes of those tumors.

Giaccia is cofounder of Ruga along with Albert Koong, who is associate professor of radiation oncology at Stanford. Ruga has five preclinical programs focused on various aspects of tumor metabolism and the cellular stress response.

Fulmer, T. SciBX 4(32); doi:10.1038/scibx.2011.893

Published online Aug. 18, 2011

\section{REFERENCES}

1. Chan, D.A. et al. Sci. Transl. Med.; published online Aug. 3, 2011; doi:10.1126/scitranslmed.3002394

Contact: Amato J. Giaccia, Stanford University School of Medicine, Palo Alto, Calif.

e-mail: giaccia@stanford.edu

2. Haase, V.H. Kidney Int. 69, 1302-1307 (2006)

3. Young, A.C. et al. Clin. Cancer Res. 15, 7582-7592 (2009)

4. Turcotte, S. et al. Cancer Cell 14, 90-102 (2008)

COMPANIES AND INSTITUTIONS MENTIONED

Cornerstone Pharmaceuticals Inc., Cranbury, N.J.

Ruga Corp., Palo Alto, Calif.

Stanford University School of Medicine, Palo Alto, Calif. 